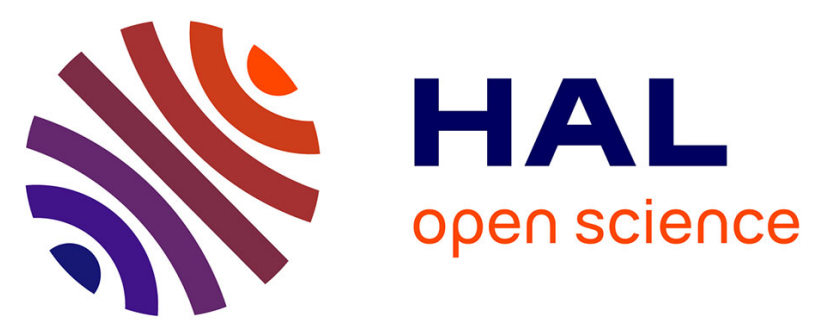

\title{
Probing the existence of uranyl trisulfate structures in the AMEX solvent extraction process
}

Tamir Sukhbaatar, Magali Duvail, Thomas Dumas, Sandrine Dourdain, Guilhem Arrachart, Pier Lorenzo Solari, Philippe Guilbaud, S Pellet-Rostaing

\section{- To cite this version:}

Tamir Sukhbaatar, Magali Duvail, Thomas Dumas, Sandrine Dourdain, Guilhem Arrachart, et al.. Probing the existence of uranyl trisulfate structures in the AMEX solvent extraction process. Chemical Communications, 2019, 55, pp.7583-7586. 10.1039/c9cc02651g • hal-02161600

\section{HAL Id: hal-02161600 \\ https://hal.umontpellier.fr/hal-02161600}

Submitted on 12 Jan 2021

HAL is a multi-disciplinary open access archive for the deposit and dissemination of scientific research documents, whether they are published or not. The documents may come from teaching and research institutions in France or abroad, or from public or private research centers.
L'archive ouverte pluridisciplinaire HAL, est destinée au dépôt et à la diffusion de documents scientifiques de niveau recherche, publiés ou non, émanant des établissements d'enseignement et de recherche français ou étrangers, des laboratoires publics ou privés. 


\section{Probing the existence of uranyl trisulfate structures in the AMEX solvent extraction process}

Tamir Sukhbaatar, ${ }^{a}$ Magali Duvail, ${ }^{\text {a }}$ Thomas Dumas, ${ }^{\mathrm{b}}$ Sandrine Dourdain, ${ }^{* a}$ Guilhem Arrachart, ${ }^{\text {a }}$ Pier Lorenzo Solari, ${ }^{\mathrm{c}}$ Philippe Guilbaud ${ }^{\mathrm{b}}$ and Stéphane Pellet-Rostaing ${ }^{\mathrm{a}}$

Knowledge of the complex microstructure in solvent extraction phases is mandatory for a full comprehension of ionic separation.

Coupling EXAFS with MD simulations for uranyl extraction in sulfuric media with tertiary amine extractants enabled unravelling of the unprecedented uranyl tri-sulfate structure.

Solvent extraction is the most widely used chemical separation method at the industrial scale. While broadly exploited, most of the processes are often not fully optimized due to the lack of understanding involved in structural and chemical mechanisms.

Amongst them, amine extraction $(\mathrm{AMEX})^{1}$ is exploited for the production and purification of uranium. Using tertiary amines as selective extracting agent enables the selective extraction of uranyl cations from sulfuric acid leach liquors, which is further exploited yearly to provide $10 \%$ of global electricity consumption. ${ }^{2}$ Considering its extensive application, the AMEX process is still broadly studied for optimisation and comprehension. However, the core mechanisms of this solvent extraction system remain poorly understood.

Conventional slope analyses have been in use since the 1950's to provide stoichiometric numbers of the extracted complexes. Coleman et al. showed that two main extraction mechanisms are involved, depending on the sulfate concentration in an aqueous phase., ${ }^{2,3}$ At low sulfate concentration, i.e., under the process conditions, adduct formation is preferred (eqn (1))

$\overline{\left(\mathrm{R}_{3} \mathrm{NH}\right)_{2} \mathrm{SO}_{4}}+\mathrm{UO}_{2} \mathrm{SO}_{4} \leftrightarrow \overline{\left(\mathrm{R}_{3} \mathrm{NH}\right)_{2} \mathrm{UO}_{2}\left(\mathrm{SO}_{4}\right)_{2}}$,

whereas anion exchange is predominant at high sulfate concentration (eqn (2))

$\overline{\left(\mathrm{R}_{3} \mathrm{NH}\right)_{2} \mathrm{SO}_{4}}+\mathrm{UO}_{2}\left(\mathrm{SO}_{4}\right)_{2}^{2-} \leftrightarrow \overline{\left(\mathrm{R}_{3} \mathrm{NH}\right)_{2} \mathrm{UO}_{2}\left(\mathrm{SO}_{4}\right)_{2}}+\mathrm{SO}_{4}^{2-}$.

In both equations the overbar refers to species present in the organic phase.

Other studies however suggested the presence of more sulfate ions in the uranium complex, without any clear explanation. McDowell et al. ${ }^{4}$ proposed four sulfates instead of two with di-n-decylamine sulfate, while Boirie, ${ }^{5}$ followed by Sato et al., ${ }^{6}$ proposed the presence of three sulfates in the uranium complex at low and high acidity

$2 \overline{\left(\mathrm{R}_{3} \mathrm{NH}\right)_{2} \mathrm{SO}_{4}}+\mathrm{UO}_{2} \mathrm{SO}_{4} \leftrightarrow \overline{\left(\mathrm{R}_{3} \mathrm{NH}\right)_{4} \mathrm{UO}_{2}\left(\mathrm{SO}_{4}\right)_{3}}$,

$4 \overline{\left(\mathrm{R}_{3} \mathrm{NH}\right) \mathrm{HSO}_{4}}+\mathrm{UO}_{2} \mathrm{SO}_{4} \leftrightarrow \overline{\left(\mathrm{R}_{3} \mathrm{NH}\right)_{4} \mathrm{UO}_{2}\left(\mathrm{SO}_{4}\right)_{3}}+2 \mathrm{H}_{2} \mathrm{SO}_{4}$.

More recently, Avelar et al. defended through a thermodynamic study that uranium is extracted as $\mathrm{UO}_{2} \mathrm{SO}_{4}$ and $\mathrm{UO}_{2}\left(\mathrm{SO}_{4}\right)_{2}{ }^{2-}$ and that the presence of $\mathrm{UO}_{2}\left(\mathrm{SO}_{4}\right)_{3}{ }^{4-}$ is unlikely under these operating conditions. ${ }^{7}$

\footnotetext{
a ICSM, CEA, CNRS, ENSCM, Univ Montpellier, Marcoule, France.

E-mail: sandrine.dourdain @ cea.fr; Fax: +334667976 11; Tel: +33466339309

${ }^{\mathrm{b}}$ CEA, DEN, MAR, DMRC, SPDS, LILA, F-30207 Bagnols-sur-Cèze Cedex, France

${ }^{c}$ Synchrotron SOLEIL, L'Orme des Merisiers, Saint-Aubin - BP 48, 91192 Gif-sur-Yvette Cedex, France

Electronic supplementary information (ESI) available: Experiments, EXAFS data acquisition and treatments, and details of MD simulations; Fig. S1 to S6 and Tables S1 to S3.
} 
As the role of sulfates appears essential in extraction mechanisms and therefore in the performance of such an extensively applied system, the aim of this study is to unravel how they bond and fit around uranyl cations.

Hennig et al. ${ }^{8}$ observed in the aqueous phase an exchange from mono- to bidendate sulfates upon increasing the sulfate concentration.

To our knowledge, with new techniques available no effort has been made in determining the uranyl coordination and structural environment in organic phases. Moreover, this process requires a phase modifier, such as octan-1ol, in order to prevent third phase formation. In all aforementioned studies, the effect of this phase modifier on the complex formation has never been looked into.

To gain an insight into the uranium extraction mechanism by tertiary amines and to figure out the essential role of sulfates, this study combines results from X-ray absorption spectroscopy with those of Molecular Dynamics (MD) calculations. This is done following an approach similar to that of Ellis et al. on different solvent extraction systems. ${ }^{9}$ The EXAFS experimental spectra interpreted using a classical fitting are compared to the recalculated spectra obtained from MD simulations.

To mimic the process conditions, the organic phases were prepared by mixing an organic solution of 0.1 molar trioctylamine and 3\% octan-1-ol in n-dodecane, with an equal volume of aqueous solution of $1 \mathrm{M}$ sulfuric acid, and $1 \mathrm{M} \mathrm{Li}_{2} \mathrm{SO}_{4}$. The uranium concentration was fixed to $10 \mathrm{mmol} \mathrm{L}^{-1}$ to optimise the EXAFS spectra signal. The detailed experimental procedure is available in the ESI, $\uparrow$ as well as the composition of the organic phases.

The uranium L3 edge EXAFS spectra and its corresponding Fourier transform recorded after solvent extraction are presented in Fig. 1.

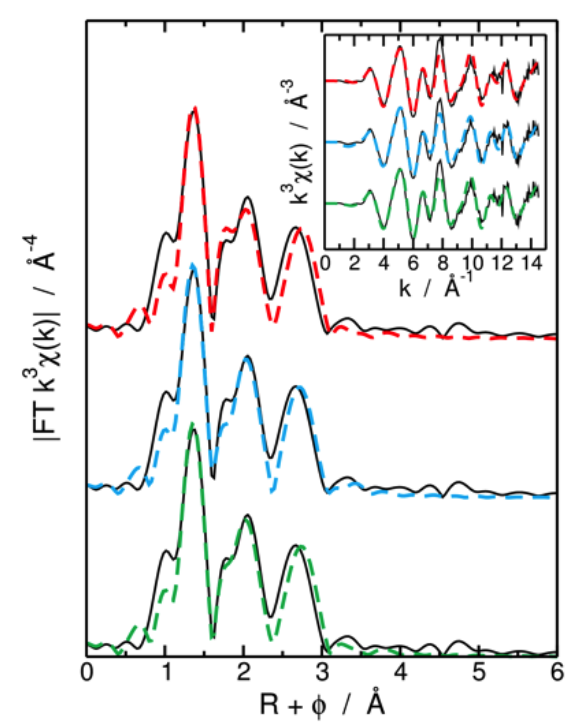

Figure 1: Experimental (full line) and fits (dashed lines) $\mathrm{k}^{3}$-weighted Fourier transformed EXAFS spectra of U-SO4-TOA complex. The green line is for the free coordination number model, the blue line is for the $2 \kappa^{2}-1 \kappa^{1} \mathrm{SO}_{4^{2-}}$ model, and the red line is for the $3 \kappa^{2} \mathrm{SO}_{4}{ }^{2-}$ model. Insert: corresponding $\mathrm{k}^{3}$-weighted EXAFS spectra.

The experimental spectra clearly differ from the typical uranyl spectra previously recorded from solvent extraction experiments: ${ }^{10-12}$ in addition to the first $\mathrm{U}-\mathrm{O}_{\mathrm{ax}}$ contribution from the linear transdioxo bonds $(\mathrm{R}+\phi=1.2 \AA)$ and to the equatorial oxygen shell $(\mathrm{R}+\phi=1.8$ to $2.4 \AA$ ) the spectra exhibit unusual intense contributions in the second coordination sphere $(\mathrm{R}+\phi>2.5 \AA$ ). Considering the extraction equilibrium (eqn (1)), this feature could be due to the sulfur atoms from the sulfate anions coordinated with $\mathrm{UO}_{2}{ }^{2+}$. Hennig et al. observed a similar contribution in aqueous solution due to the sulfate anion coordinated with uranyl. ${ }^{13}$ Depending on the sulfate coordination mode, this contribution shifts from $\mathrm{R}+\phi=2.6 \AA$ to $3 \AA$ from bidentate $\left(\mathrm{k}^{2}\right)$ to monodentate $\left(\mathrm{k}^{1}\right)$ sulfate, respectively. Nevertheless, in aqueous media and under concentrated sulfate conditions ( 2 molar), the sulfate coordination number is limited to 2 . In this work, the sulfur atom signal isintense and differs from previous reports of EXAFS. In order to probe the uranyl local structure, a fit of the EXAFS spectra was obtained by applying different a priori structural models. Three models providing the best fit are summarised in Table 1. 
Table 1: Structural parameters for 3 bidentate, and 2 bi- and 1 monodentate sulfates coordinated to an uranyl cation.

\begin{tabular}{|c|c|c|c|c|c|c|c|c|c|}
\hline \multirow[b]{2}{*}{ Path } & \multicolumn{3}{|c|}{$2 \kappa^{2}-1 \kappa^{1} \mathrm{SO}_{4}^{2-}(\mathrm{a})$} & \multicolumn{3}{|c|}{$3 \kappa^{2} \mathrm{SO}_{4}^{2-}(\mathrm{b})$} & \multicolumn{3}{|c|}{ Floating(c) } \\
\hline & $N$ & $R / \AA$ & $\sigma^{2} / \AA^{2}$ & $N$ & $R / \AA$ & $\sigma^{2} / \AA^{2}$ & $N$ & $R / \AA$ & $\sigma^{2} / \AA^{2}$ \\
\hline$\overline{\mathrm{U}-\mathrm{O} y l}$ & $2(*)$ & $1.78(1)$ & $0.0028(18)$ & $2(*)$ & $1.77(1)$ & $0.0012(13)$ & $2(*)$ & $1.78(1)$ & $0.0019(16)$ \\
\hline U-Oeqmono & $1(*)$ & $2.25(9)$ & $0.0065(51)$ & & & & $0.3(2)$ & $2.21(1)$ & $0.0020(5)$ \\
\hline $\mathrm{U}-\mathrm{O} e q b i$ & $4(*)$ & $2.45(2)$ & $0.0039(24)$ & $6(*)$ & $2.46(2)$ & $0.0062(24)$ & $5.4(4)$ & $2.45(2)$ & $0.0060(14)$ \\
\hline U-Smono & $1(*)$ & $3.90(19)$ & $0.0099(125)$ & & & & $0.3(2)$ & $3.99(72)$ & $0.0061(44)$ \\
\hline $\mathrm{U}-\mathrm{S} b i$ & $2(*)$ & $3.14(2)$ & $0.0022(24)$ & $3(*)$ & $3.16(2)$ & $0.0029(18)$ & $2.7(2)$ & $3.11(2)$ & $0.0031(15)$ \\
\hline
\end{tabular}

Overall, best-fits are obtained from models three sulfate anions in the uranyl coordination sphere.

This structure confirms the uranyl/sulfate ratio of $1 / 3$ ratio suggested by Boirie ${ }^{5}$ and Sato et al. ${ }^{6}$ from the slope method.

This is also in agreement with the structure proposed by Doran et al. by a crystallographic study on uranium sulfates stabilized by damines. ${ }^{14}$

To estimate the hypothetical effect of coordinating water and/or octan-1-ol molecules on the EXAFS fit, further fit models are made with a third $\mathrm{O}$ atom shell into the uranyl equatorial coordination sphere. The experimental spectra and their fits obtained with a varying number of water and/or octan-1-ol molecules (0 to 2) are shown in Fig. S2 (ESI $\dagger$ ). It is shown that, considering the very intense sulfur atom contribution in the experimental spectra, the best fit is obtained from the first coordination shell containing exclusively sulfate ligands.

Hence, EXAFS fitting demonstrates that neither octan-1-ol nor water is present in the first coordination sphere of uranyl. For $1 / 3$ uranyl/sulfate stoichiometry, the experimental spectra can be equivalently fitted using three structural models: two models with fixed coordination numbers including either three bidentate sulfate $\left(3 \mathrm{k}^{2}\right)$ or two bidentate plus one monodentate $\left(2 \mathrm{k}^{2}-1 \mathrm{k}^{1}\right)$ and one intermediate model set with a floating number of bi- or monodentate sulfate ligands $\left(\mathrm{k}^{2} \leftrightarrow \mathrm{k}^{1}\right)$. They also provide good results with 2.7 bidentate and 0.3 monodentate sulfates. However, besides small changes in the Debye-Waller factor values $\left(\sigma^{2}\right)$ and in the amplitude reduction factor $\left(\mathrm{S}_{0}^{2}\right)$ which in turn balances changes in the coordination numbers, the three models are statistically equivalent. From such a classical fit approach, it is impossible to undoubtedly assign a predominant structure for the uranyl trisulfate complex.

Only the $2 \mathrm{k}^{2}-1 \mathrm{k}^{1} \mathrm{SO}_{4}{ }^{2-}$ coordination mode for which a doubtful $\sigma^{2}$ value for the bidentate sulfate is obtained $\left(0.0022 \AA^{2}\right)$ lower than the $\mathrm{O}_{\mathrm{yl}} \sigma^{2}\left(0.0028 \AA^{2}\right)$ could eventually be ignored. The uranyl trisulfate complex seems to switch from its main form - the 3 bidentate sulfate configuration to the 2 bidentate and 1 monodentate configuration. To precisely determine the complex structure, we applied a molecular dynamic analysis taking into consideration the dynamic changes in the ligand coordination mode.

In an attempt to obtain clear details on the exact structure of uranyl in regard to its $\mathrm{SO}_{4}{ }^{2-}$ coordination, classical molecular dynamics (MD) simulations of one uranyl cation complexed by three sulfate anions interacting with 4 protonated tri-n-octylamine molecules have been performed in n-dodecane using explicit polarization. In line with experimental hypothesesmade regarding the very low water content measured in the organic phase

(Table S1, ESI $\dagger$ ) and the presence of only sulfate anions in the $\mathrm{UO}_{2}{ }^{2+}$ first coordination shell (Fig. S1, ESI $\dagger$ ), neither water nor the octan-1-ol molecule have been considered in complex simulation.

Simulation details are provided in the ESI. $\dagger$ The radial distribution functions calculated from MD simulations provided the structural properties of $\mathrm{UO}_{2}{ }^{2+}$ (Fig. S5, ESI $\dagger$ ).

The distances and Debye-Waller factors calculated from MD simulations are provided in Table 2. 
Table 2: Structural parameters calculated from MD simulations

\begin{tabular}{lccc}
\hline Path & $N$ & $R / \AA$ & $2 \sigma^{2}$ \\
\hline U-Oyl & 2 & $1.78 \pm 0.01$ & $0.0010 \pm 0.0001$ \\
U-Oeqmono & $2.5 \pm 0.7$ & $2.53 \pm 0.02$ & $0.0081 \pm 0.0015$ \\
U-Oeqbi & $3.1 \pm 0.7$ & $2.43 \pm 0.01$ & $0.0034 \pm 0.0003$ \\
U-Oeqaverage & 5.6 & $2.46 \pm 0.01$ & $0.0064 \pm 0.0002$ \\
U-Smono & $0.3 \pm 0.01$ & $3.44 \pm 0.01$ & $0.0269 \pm 0.0037$ \\
U-Sbi & $2.7 \pm 0.02$ & $3.10 \pm 0.01$ & $0.0062 \pm 0.0001$ \\
\hline
\end{tabular}

The Debye-Waller factors and distances calculated from MD simulations are in good agreement with those obtained by using the classical EXAFS fitting procedure. This is specifically true for the experimental fit performed using the $3 \mathrm{k}^{2} \mathrm{SO}_{4}{ }^{2-}$ model.

Indeed, small U-O $\mathrm{O}_{\text {eq }}$ distances (2.21-2.25 $\AA$ ) for monodentate coordination and large U-S monodentate distances (3.90-3.99 $\AA$ ) determined from the experimental fits considering the $2 \mathrm{k}^{2}-1 \mathrm{k}^{1} \mathrm{SO}_{4}{ }^{2-}$ model or from the free coordination model are not found using MD simulations (Fig. S5, ESI $\dagger$ ). It can also be noticed here that fitting the $\mathrm{U}-\mathrm{O}_{\mathrm{eq}}$ distance considering two types of oxygen atoms (bidentate vs. monodentate) did not give satisfactory results since the ratio between both coordinations differs considering either the oxygen atoms or the sulfur atoms. Indeed, MD reflects only average coordination number of oxygen atoms and is not able to differentiate between equivalent distances. However, MD simulations clearly point out an equilibrium between aforementioned two configurations, i.e., 3 bidentate sulfate $\left(3 \mathrm{k}^{2} \mathrm{SO}_{4}{ }^{2-}\right)$ vs. 2 bidentate and 1 monodentate $\left(2 \mathrm{k}^{2}-1 \mathrm{k}^{-\mathrm{SO}_{4}}{ }^{2-}\right)$ (Fig. 2$)$.
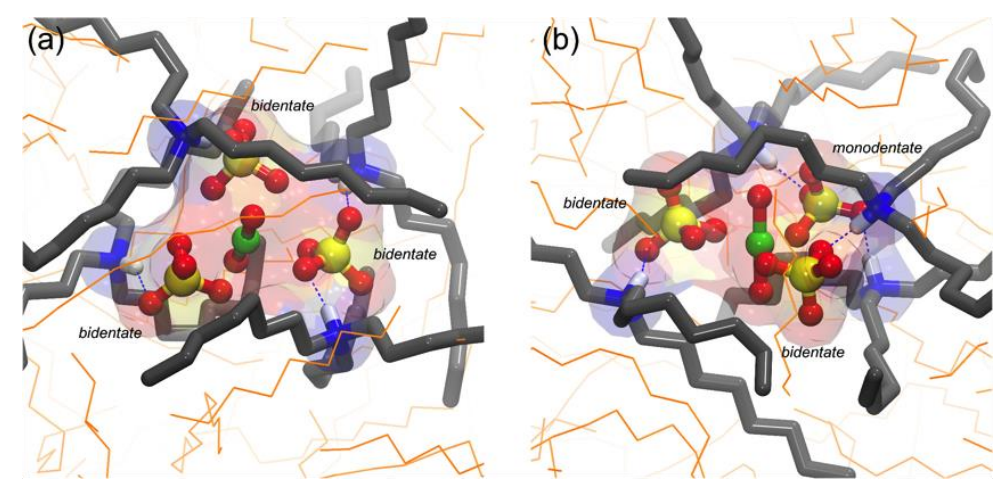

Figure 2: Snapshots issued from MD simulations performed in $n$-dodecane presenting the complex $\mathrm{UO}_{2}\left(\mathrm{SO}_{4}\right)_{3}(\mathrm{TOA})_{4}$ with (a) $3 \mathrm{SO}_{4}{ }^{2-}$ in the bidendate coordination, and (b) 2 bidentate and 1 monodentate $\mathrm{SO}_{4}{ }^{2-}$. The uranium atom is coloured in green, oxygen atoms are in red, sulfur atoms are in yellow, nitrogen atoms are in blue, carbon atoms are in black, and hydrogen atoms are in white ( $\mathrm{H}$ atoms bonded to carbon atom are not represented). $n$-dodecane molecules are coloured in orange.

The structural properties calculated using MD simulations show that the $3 \mathrm{k}^{2} \mathrm{SO}_{4}{ }^{2-}$ configuration seems to be preferred compared to $2 \mathrm{k}^{2}-1 \mathrm{k}^{1}$ since the latter occurred for $10 \%$ of the total simulation time only. Unfortunately, when considering only an average stoichiometry of the complex, no clear prediction on the coordination mode of $\mathrm{SO}_{4}{ }^{2-}$ in the $\mathrm{UO}_{2}{ }^{2+}$ first coordination shell was made. From MD simulations, we exported as a function of time all $3 \mathrm{k}^{2}$ and $2 \mathrm{k}^{2}-1 \mathrm{k}^{1} \mathrm{SO}_{4}{ }^{2-}$ configurations and analysed separately both types of configurations. Fourier transforms and EXAFS spectra have been calculated without any fitting parameter for both configurations.

The three theoretical EXAFS spectra and associated Fourier transforms, i.e., for the entire MD simulation, and for the $2 \mathrm{k}^{2}-1 \mathrm{k}^{1}$ and the $3 \mathrm{k}^{2}$ configurations, have been compared to the experimental EXAFS signal and Fourier transform (Fig. 3). 


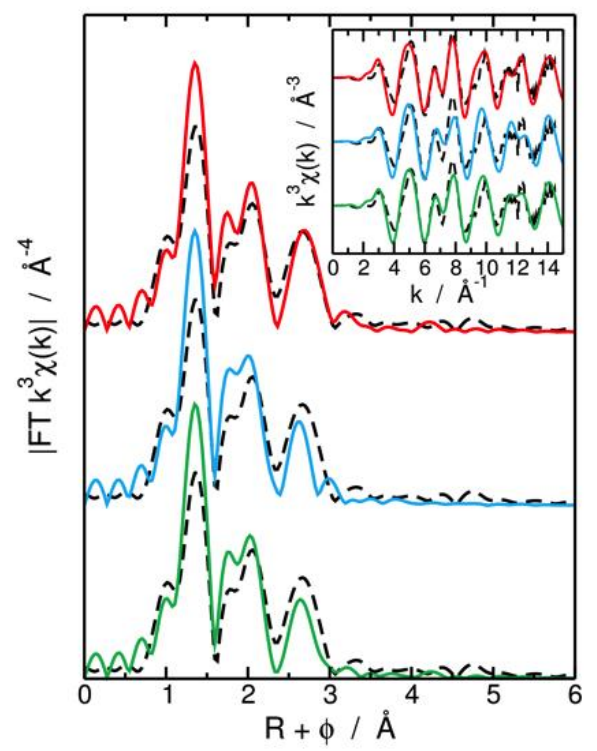

Figure 3: $\mathrm{k}^{3}$-weighted Fourier transformed (FT) EXAFS spectra obtained for a $\mathrm{k}$ range of 3 to $14.5 \AA^{-1}$ determined experimentally (black dashed line) and calculated from MD simulations for the whole MD simulation (green solid line), for the $2 \mathrm{k}^{2}-1 \mathrm{k}^{1} \mathrm{SO}_{4}{ }^{2-}$ configurations (blue solid line) and for the $3 \mathrm{\kappa}^{2} \mathrm{SO}_{4^{2-}}$ configurations (red solid line). Corresponding $\mathrm{k}^{3}-$ weighted EXAFS spectra are presented in the insert.

It can be noticed here that high theoretical intensities of the FT peak located at $\mathrm{R}+\phi=1.3 \AA$ are originated from the very rigid U-Oyl bending used in MD simulations. ${ }^{15}$ This comparison clearly shows that the average EXAFS signal and Fourier transform calculated taking into consideration only $3 \mathrm{k}^{2}$ configurations from MD simulation fits best the experimental ones observed for the EXAFS fitting procedure.

A more precise analysis of the interaction between TOA molecules and sulfate anions in MD simulations provides evidence of the interdependence between the sulfate coordination mode to uranyl and H-bond formation (between the sulfate anions and the protonated amine function). Indeed, changes in the $\mathrm{U}-\mathrm{N}_{\mathrm{TOA}}$ (or $\mathrm{U}-\mathrm{H}_{\mathrm{TOA}}$ ) distances are observed during $\mathrm{k}^{2} \leftrightarrow \mathrm{k}^{1}$ exchange of $\mathrm{SO}_{4}{ }^{2-}$ anions (Fig. S6, ESI $\dagger$ ). This has also been confirmed by a crystallographic study of Doran et al. ${ }^{14}$ This dynamic behavior suggests that uranyl sulfate complex extraction is driven by different sulfate-amine interactions, as proposed by Williams et al. ${ }^{16}$ which may also be affected by slight changes in the organic phase water content. Hence, the nature of the diluent or other parameters that could modify the water content in the organic phase may drastically change the H-bond interactions and therefore the stability of the two $\left[\mathrm{UO}_{2}\left(\mathrm{SO}_{4}\right)_{3}\right]^{4-}$ configurations identified in this experimental project. This approach paves the way for uranyl extraction process optimization with focus on weak H-bond interactions and, indirectly, the control of the extraction mechanism via molecular aggregation and organization in organic phases.

With respect to the conditions of the study, both MD and classical fitting approaches led to the same speciation in the organic phase: three sulfate anions coordinated with uranyl cations through $\mathrm{U}-\mathrm{O}$ bonds in the first coordination shell. The EXAFS spectra show that uranyl trisulfate complexes switch between 3 bidentate sulfate configuration, and 2 bidentate and 1 monodentate configuration.MD simulations confirmed this result and proved that three bidentate sulfate anions are more favorable than 2 bidentates and 1 monodendate. In addition to this sulfate coordination aspect, the exchangeable hydrogen bond distribution observed by MD indicates a strong interrelation between the inner- and outer-sphere complex environment. This behavior confirms the importance of weak association on the molecular organization of an organic phase and also to the uranyl coordination as previously demonstrated by other solvent extraction systems. ${ }^{17,18}$ EXAFS analysis showed that octan-1-ol and water do not penetrate the first coordination sphere. However, this raises questions on the potential role of outer sphere molecules (water, octan-1-ol or diluent) in supramolecular and molecular architectures and their secondary impact on the first coordination spheres.

This study paves the way for better understanding of weak association and diluent effects on solvent extraction mechanisms. 


\section{Acknowledgements}

This work was supported by the program NEEDS Resources.

We also acknowledge Ms Ngobay for fruitful discussion and help in the writing of this manuscript.

\section{Conflicts of interest}

There are no conflicts to declare.

\section{Notes and references}

1 D. Crouse, K. Brown and W. Arnold, Progress Report on Separation and Recovery of Uranium and Thorium from Sulfate Liquors by the AMEX Process, Oak Ridge National Lab., Tenn., USA, 1957.

2 C. F. Coleman, K.B. Brown, J. G. Moore and D. J.Crouse, Ind. Eng. Chem., 1958, 50, 1756-1762.

3 C. F. Coleman, Nucl. Sci. Eng., 1963, 17, 274-286.

4 W. J. McDowell and C. F. Baes, J. Phys. Chem., 1958, 62(7), 777-783.

5 C. Boirie, Bull. Soc. Chim. Fr., 1958, 8-9, 1088-1093.

6 T. Sato, J. Inorg. Nucl. Chem., 1963, 25, 441-446.

7 E. C. Avelar, C. L. G. Alvarenga, G. P. S. Resende, C. A. Morais and M. B. Mansur, Brazilian J. Chem. Eng., 2017, 34, 355362.

8 C. Hennig, K. Schmeide, V. Brendler, H. Moll, S. Tsushima and A. C. Scheinost, Inorg. Chem., 2007, 46, 5882-5892.

9 R. J. Ellis, B. Reinhart, N. J. Williams, B. A.Moyer and V. S. Bryantsev, Chem. Commun., 2017, 53, 5610-5613.

10 O. Pecheur, S. Dourdain, D. Guillaumont, J. Rey, P. Guilbaud, L. Berthon, M. Charbonnel, S. Pellet-Rostaing and F. Testard, J. Phys. Chem. B, 2016, 120, 2814-2823.

11 E. Acher, Y. H. Cherkaski, T. Dumas, C. Tamain, D. Guillaumont, N. Boubals, G. Javierre, C. Hennig, P. L. Solari and M.C. Charbonnel, Inorg. Chem., 2016, 55, 5558-5569.

12 Q. Sun, B. Aguila, J. Perman, A. S. Ivanov, V. S. Bryantsev, L. D. Earl, C. W. Abney, L. Wojtas and S. Ma, Nat. Commun., 2018, 9, 1644.

13 C. Hennig, W. Kraus, F. Emmerling, A. Ikeda and A. C. Scheinost, Inorg. Chem., 2008, 47, 1634-1638.

14 M. B. Doran, A. J. Norquist and D. O'Hare, Inorg. Chem., 2003, 42, 6989-6995.

15 M. Duvail, T. Dumas, A. Paquet, A. Coste, L. Berthon and P. Guilbaud, Phys. Chem. Chem. Phys., 2019, 21, $7894-7906$.

16 N. J. Williams, C. A. Seipp, K. A. Garrabrant, R. Custelcean, E. Holguin, J. K. Keum, R. J. Ellis and B. A. Moyer, Chem. Commun., 2018, 54, 0048-10051.

17 J. R. Turkington, P. J. Bailey, J. B. Love, A. M. Wilson and P. A. Tasker, Chem. Commun., 2013, 49, 1891-1899.

18 A. M. Wilson, P. J. Bailey, P. A. Tasker, J. R. Turkington, R. A. Grant and J. B. Love, Chem. Soc. Rev., 2014, 43, $123-134$. 